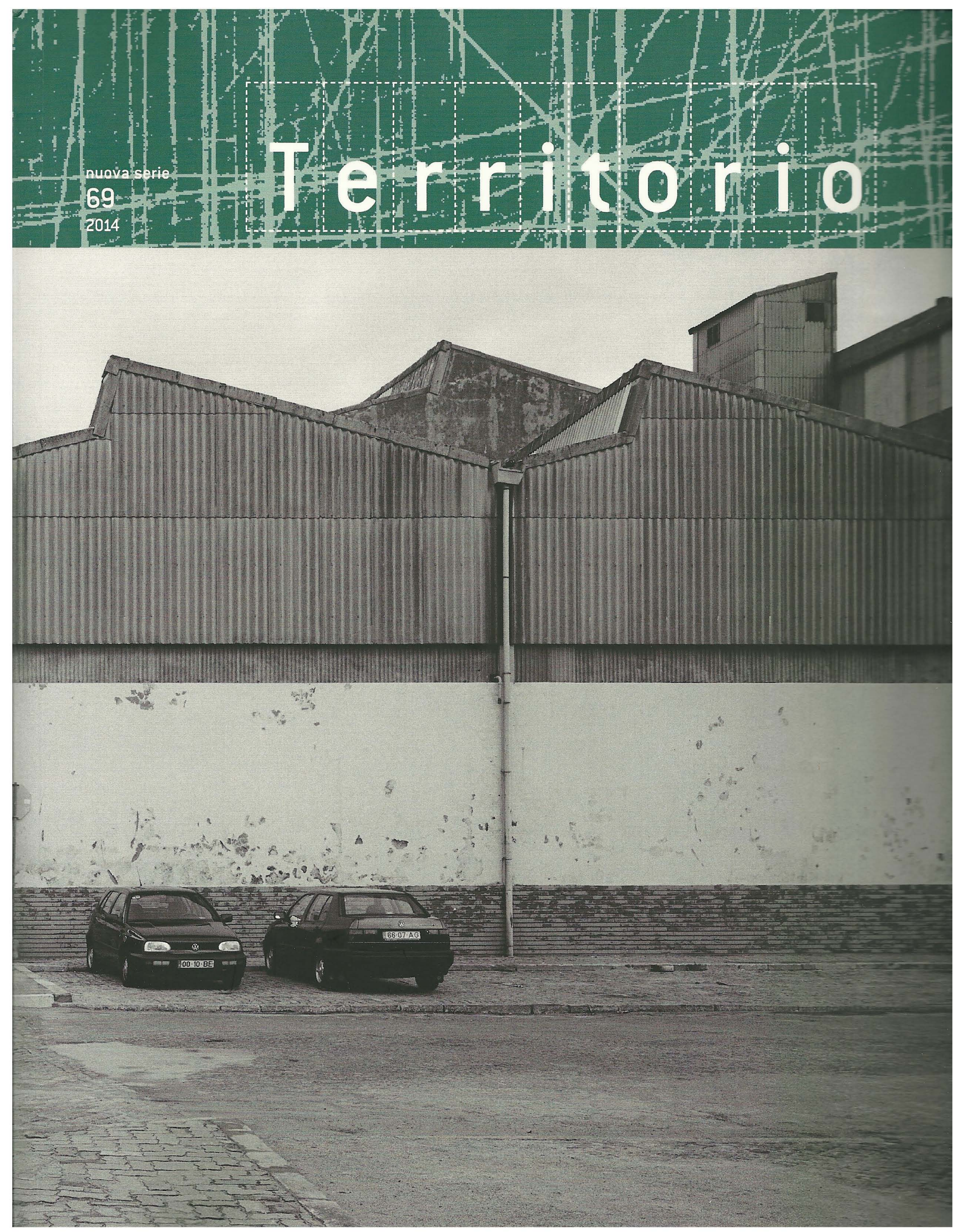




\section{Transbordering planning: esempi dalle Americhe Latine ${ }^{1}$}

Clara Irazábal

Graduate School of Architecture, Planning and Preservation, Columbia University, New York

(cei2108@columbia.edu)

\begin{abstract}
Le discipline spaziali sono chiamate ad interrogarsi con maggiore attenzione sul ruolo che le dinamiche transnazionali hanno nella configurazione del paesaggio contemporaneo, nelle sue forme urbane, sociali ed identitarie. Lo studio di processi transnazionali nel contesto latino-americano, all'interno $e$ al di là delle sue frontiere politiche, sociali, culturali e simboliche, mostra come le popolazioni migranti trascendano, negozino e ridisegnino i confini dei regimi di potere esistenti, attraverso le proprie pratiche abitative e di mobilità a diverse scale. Transbordering Latin Americas, ultimo progetto del Latin Lab della Columbia University, presenta una serie di ricerche che ragionano sulla diffusione globale d'immaginari, modi d'abitare e luoghi latino-americani che riflettono e, al contempo, contestano le dinamiche transnazionali del capitalismo globale
\end{abstract}

Parole chiave: processi urbani transnazionali: transbordering planning; pratiche migranti
Si sta diffondendo in tutto il mondo una crescente consapevolezza dell'importanza di comprendere e seguire le dinamiche transnazionali nella pratica e nell'insegnamento della pianificazione. Se il mantra «la pianificazione è sempre locale», spesso ripetuto da professionisti, studiosi e da istituzioni internazionali come UN-Habitat, rimane ancora profondamente vero, è altrettanto vero che il 'locale' possiede sempre di più una dimensione multi-scalare. Le conurbazioni che oltrepassano i limiti comunali, a volte persino i confini regionali (di distretto, provinciali, statali) o nazionali, richiedono un approccio innovativo da parte della pianificazione e delle politiche urbane. Nonostante i tentativi degli studiosi di pianificazione di tener conto di questi fenomeni emergenti a livello globale, rimaniamo ancora in ritardo rispetto alla realtà, visto che non possiedendo ancora un linguaggio adeguato per poter nominare o spiegare le modalità con cui le persone vivono in un mondo transnazionale, e tantomeno i mutamenti sociali e gli insediamenti spaziali che esse producono.

Poiché non possediamo una conoscenza approfondita di tali questioni, sono stati fatti pochi progressi nell'ideare nuovi strumenti istituzionali e gestionali per regolare queste nuove realtà insediative, variamente etichettate come città-regioni, megalopoli, cosmopoli, città globali, ethnoscapes ${ }^{3}$, :ecc. Tra le altre scienze, la nostra disciplina dovrebbe dedicare maggiori sforzi nel comprendere i diversi sotto-ambiti della pianificazione regionale e transnazionale; le implicazioni socio-politiche, culturali e spaziali dei regimi transfrontalieri; e la sfida sia a costruire un'infrastruttura sociale e fisica, sia a diminuire le diseguaglianze tra luoghi e comunità divise da frontiere spaziali, materiali e immateriali. Gli studiosi di pianificazione dovrebbero, inoltre, valutare costantemente l'efficacia individuale, collettiva, istituzionale e socio-spaziale degli attori coinvolti in queste pratiche transnazionali e proporre riforme rilevanti o innovative in relazione a politiche, programmi e progetti che possano dare luogo a città più giuste e sostenibili.

Insieme a queste dinamiche che avvengono sia attraverso che a causa delle frontiere, la pianificazione dovrebbe porre maggiore attenzione agli effetti dei processi migratori ed etnici sul paesaggio urbano. La produzione dello spazio urbano, del potere, della conoscenza e delle soggettività in determinati luoghi è strettamente legata a processi che avvengono altrove. I movimenti di persone, capitale, lavoro, turismo, informazione, media e idee legate alla progettazione urbana e alla pianificazione trascendono le frontiere delle nazioni e gli immaginari collettivi dei loro 
popoli. Gli studiosi di pianificazione dovrebbero dedicarsi maggiormente allo studio dei fattori attrattivi e repulsivi che danno luogo alle migrazioni; le dinamiche socio-spaziali, culturali e politiche dei luoghi di origine, di destinazione e di transito; e l'impatto della loro reciproca sovrapposizione nella creazione dei luoghi (Irazábal, 2011). Nel fare questo, la pianificazione dovrebbe ambire a disvelare e promuovere spazi di nuova speranza all'interno di queste forme di mobilità, soprattutto per coloro i quali sono privati dei diritti fondamentali a causa della loro etnia. Ritengo che tali dinamiche, complesse, interrelate e in continua espansione, costituiscano alcune delle maggiori sfide per la pianificazione nel nostro secolo. Per questa ragione, il Latin American and Caribbean Laboratory (Latin Lab) che dirigo presso la Columbia University sta dedicando particolare attenzione all'analisi di tali questioni ${ }^{4}$. L'ultima pubblicazione da me curata, Transbordering Latin Americas: Liminal Places, Cultures, and Powers (T)Here, è un esempio dei progetti coordinati dal Laboratorio. Il libro rappresenta il più recente lavoro del Latin Lab ed esamina le interconnessioni tra urbanizzazione e migrazioni, sia nel generare sia nel riflettere la riorganizzazione globale di processi di produzione e riproduzione sociale attorno a soggetti e gruppi latino-americani, in tutto il mondo ${ }^{5}$. Obiettivo del volume è di aiutarci a comprendere una serie di fenomeni che si stanno dispiegando davanti ai nostri occhi e a individuare modalità di intervento, politiche ed azioni che possano assisterci nel costruire competenze in una prospettiva di cambiamento progressista. Il libro esamina le condizioni socio-culturali e spaziali attraverso e al di là dalle frontiere dell'America Latina, in tutto il globo e a differenti scale, che vanno da singoli soggetti interessati da discriminazioni di genere e razza, fino a organizzazioni nazionali e transnazionali. Riunendo studiosi delle 'scienze spaziali' - architettura, urban design, urbanistica e geografia - come anche sociologia, antropologia, storia ed economia, il volume esplora queste pratiche di produzione di luoghi e di comunità attraverso confini culturali e stato-nazionali, esaminando differenti soggetti (individui, gruppi etnici e culturali, Ong, progettisti, operatori economici, e agenzie governative) coinvolti in pratiche abitative transnazionali/transfrontaliere e di produzione dell'ambiente urbano, riconsiderando le nozioni di stato, identità e cittadinanza. Gli studi mostrano, inoltre, come popolazioni assoggettate resistano, si adattino e co-producano progetti transnazionali/ transfrontalieri e, nel processo, aiutino a formare e al contempo siano plasmati in quanto soggetti attivi attraverso le frontiere. Nel saggio che segue, basato su questo nostro recente lavoro (Irazábal, 2014), ho voluto offrire a Territorio una delle sue stimolanti 'Aperture', cercando di presentare nuove idee e suscitare nuovi dibattiti tra studiosi dell'urbano in generale, e pianificatori, urbanisti e architetti, in particolare.

Transbordering Planning: cosa significa Transbordering? Il termine transbordering, che qui propongo, coglie le sfumature del concetto di transnazionalismo e, allo stesso tempo, ne supera le limitazioni, come spiegherò in questa sezione. Nozione multidisciplinare, il termine transnazionale ha attratto l'attenzione di quegli studiosi che ritenevano concetti come internazionale, globalizzazione o cosmopolitismo, troppo rigidi per definire le sfumature complesse e mutevoli del mondo contemporaneo. Trans è un prefisso che significa sopra, al di là o oltre. In un mondo fatto d'interconnessioni, le storie personali e le pratiche di molti individui e comunità spesso oltrepassano i confini di una particolare cultura o località all'interno degli stati nazionali, destabilizzando le preesistenti condizioni geografiche e di potere.

Transnazionalismo indica, da un lato, quelle reti (di relazioni) trans-frontaliere sviluppate da comunità localizzate che, in maniera abituale, viaggiano o si connettono ad altre persone all'estero e, dall'altro, le modalità attraverso cui tali reti collegano geograficamente luoghi distinti in singoli ambiti sociali (Trotz, 2006). Le nozioni di inter-, sovra-, ri-, e post-nazionalizzazione come anche di globalizzazione, glocalizzazione, diaspora, e trans-nazionalizzazione hanno contribuito ad una più complessa comprensione della nascita e delle dinamiche di questi spazi sociali densi e vibranti (Pries, 2005), ma hanno anche fallito sotto alcuni aspetti. Le pratiche sociali transnazionali sono rese uniche dal fatto che esse includono molteplici spazi di localizzazione $e$ articolazione (Smith, 2001), spazi di luoghi e spazi di flussi (Castells, 2004) che oltrepassano un singolo stato-nazione. Allo stesso tempo, il transnazionalismo non riguarda solo ed esclusivamente il movimento: il movimento, almeno delle persone, non è un prerequisito per impegnarsi in pratiche transnazionali (Levitt, 2001).

Il termine transnazionalismo è usato per riferirsi alle «specificità culturali dei processi globali» (Ong, 1999, p. 4) e alle molteplici dimensioni delle pratiche di produzione dei luoghi che trascendono gli stati-nazione. Il concetto fu utilizzato per la prima volta in letteratura economica in riferimento al movimento di capitale, alle catene di produzione e all'impatto delle compagnie multinazionali. È stato in seguito esteso ai flussi migratori internazionali e al loro ruolo nell'accrescere i legami transfrontalieri attraverso visite di ritorno e rimesse (per una genealogia del termine si veda Duany, 2011). Se da un lato il transnazionalismo rimodella le realtà locali, dall'altro le pratiche transnazionali sono mediate a loro volta da fattori locali, sebbene questi ultimi siano stati studiati in misura molto minore (Portes, Guarnizo, and Landolt, 1999; Torres and Momsen, 2005). Gli esempi riportati in questo saggio intendono rimediare a queste ed altre limitazioni.

Il fondamentale testo di Michael Peter Smith (2001, p. 5) sull'urbanistica transnazionale la definisce «una metafora culturale piuttosto che strettamente geografica». Smith articola, inoltre, una distinzione concettuale tra globalizzazione e transnazionalismo. I discorsi relativi a globalizzazione e transnazionalismo si differenziano per le ipotesi che essi fanno riguardo il ruolo dello stato nella produzione e negoziazione di potere, conoscenza, soggettività e spazio che, a loro volta, danno forma a significati, identità e relazioni sociali (Irazábal, 2009). Il transnazionalismo, poiché differente dalla globalizzazione, coglie la natura orizzontale e relazionale dei processi economici, sociali e culturali contemporanei che fluiscono attraverso gli spazi (Smith, 2001). Esso esprime tali processi di radicamento in scale e regimi di potere differentemente configurate e riconfigurate (Irazábal, 2005; Ong, 1999).

Nel dibattito sull'architettura e sulla città, il transnazionalismo/ transborderism fornisce un quadro di riferimento grazie al quale affrontare un cambiamento della società e della professione nella costruzione del lungo, per mezzo del quale le conoscenze geografiche tradizionali vengono problematizzate e riviste 


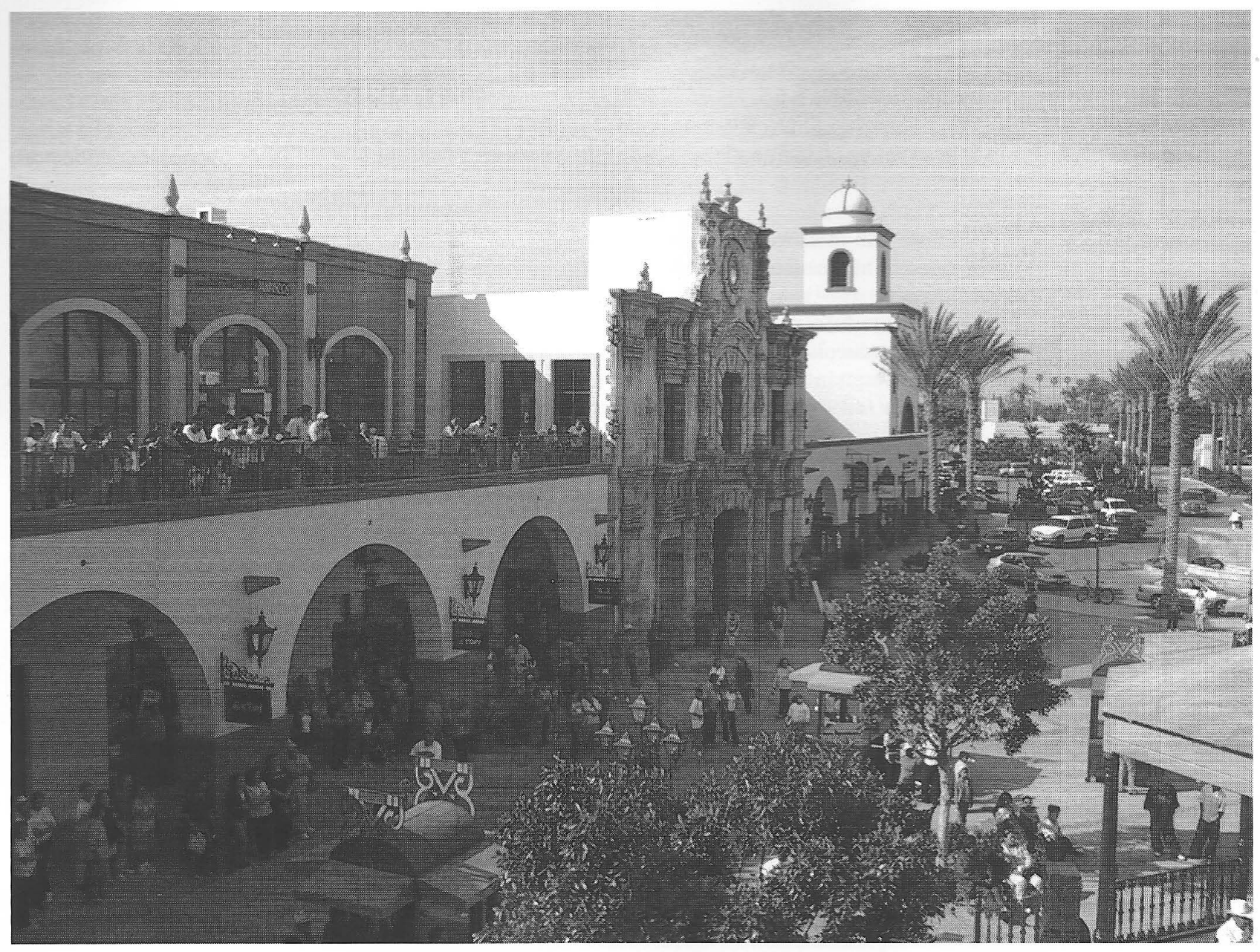

Veduta di Plaza Mexico (Los Angeles, Usa). La copia della facciata del Palazzo del Governo di Jalisco intende dare l'impressione di essere in Messico

Fonte: foto di G. Fumero 
per poter svolgere nuovi ruoli nello sviluppo di uno spazio socialmente costruito. Smith afferma: «Lo stato-nazione e le pratiche transnazionali non dovrebbero essere mutualmente esclusive. In questo processo, nazione e stato richiederebbero di essere attentamente svincolati, dando spazio a nazionalismi de-territorializzati (ovvero slegati dai loro vincoli all'indissolubile unità dello stato territoriale) e legandosi sia al livello locale che trans-locale» (Smith, 2001, p. 4).

I teorici del transnazionalismo trattano lo stato-nazione e le pratiche transnazionali come formazioni sociali interconnesse, sovrapposte e mutualmente costitutive, dove vengono prodotte e riprodotte forme di identità. Appadurai riconosce le speciali 'trans-località' che questi processi producono, "nei quali i legami di matrimonio, lavoro, affari e svago intrecciano insieme varie popolazioni in movimento con (diversi) tipi di luoghi» (Appadurai, 1996, p. 192).

La concezione di Jackson, Crang e Dwyer degli spazi della transnazionalità include: «non solo le geografie materiali della manodopera dei migranti o il commercio di beni e servizi transnazionali, ma anche le geografie simboliche e immaginarie attraverso le quali cerchiamo di dare senso ad un mondo sempre più transnazionale. Lo spazio transnazionale è, noi riteniamo, complesso, multidimensionale e abitato in maniere diverse (Crang et al., 2003). Le persone, da vari contesti, accedono a questi spazi con una ampia gamma di investimenti e da varie condizioni posizionali. Costoro possono occupare questi spazi momentaneamente (durante il consumo di un pasto, per esempio) oppure per tutta la vita (come i membri di comunità transnazionali etnicamente definite). Possono avere affinità residuali con le identità transnazionali di migranti di generazioni precedenti o identità emergenti derivanti dalle loro attuali esperienze transnazionali. Concentrarsi sugli spazi della transnazionalità, piuttosto che semplicemente su comunità transnazionali identificabili e distinte da altre comunità nazionali (spesso su base normativa), apre a modalità (nuove) di esplorazione della molteplicità di relazioni ed esperienze transnazionali» (Jackson, Crang and Dwyer, 2004, p. 1; cit. in Torres and Momsenm 2005, p. 319).

Nonostante le numerose modalità con cui sono stati teorizzati, i termini transnazionale - con riferimento ad una entità politica precisa, ossia lo stato nazionale - e translocale - con il suo legame ad una società fondata in un luogo - non colgono pienamente i tanti confini che i migranti ed altre persone attraversano. Stephen ha posto la questione riferendosi ai migranti messicani, «Le frontiere che essi attraversano in Messico sono etniche, di classe, culturali, coloniali, e statali, come anche presso la frontiera tra Messico e Stati Uniti e in differenti regioni degli Usa. ... Attraversare le frontiere nazionali è un tipo di attraversamento ... ma ve ne sono molti altri ancora" (Stephen, 2007, p. 6). Perciò, benché abbiano un impatto molteplice sulle vite delle società attraverso i confini nazionali, le esperienze transnazionali sono meglio definite "come un sotto-insieme di un approccio olistico alle esperienze transfrontaliere» (Bada, 2010 , p. 243). Nella sua espressione verbale, transbordering, invece che transnazionalismo, meglio allude agli attuali aspetti trasversali, transazionali, traslazionali e, a volte, trasgressivi dei comportamenti e degli immaginari contemporanei che sono promossi, attivati e regolati dalle logiche degli stati, delle società, dalle tensioni tra capitalismo/anti-capitalismo a differenti scale, al di sopra e al di sotto dello stato-nazione (Irazábal, 2005). Se le frontiere sono dettate dalle politiche d'inclusione ed esclusione, presidiate non solo alle frontiere fisiche, ma anche negli spazi pubblici, nei luoghi di lavoro, nello spazio domestico e nel corpo (Bauder, 2013), le pratiche che hanno luogo attraverso e a causa delle frontiere alludono all'azione sia d'individui sia di gruppi che negoziano ed interrompono le relazioni di potere egemoniche (esistenti) per migliorare le proprie condizioni di vita. Molte di queste interferenze non hanno una trascendenza politica, ma alcune possono divenire riformiste o addirittura rivoluzionarie (Irazábal, 2008).

In questo modo, proprio come il termine transnazionale trova la sua radice epistemologica nello stato-nazione e il termine trans-locale nel locale, entrambi superano le proprie origini; la nozione di transbordering indica e si allontana, allo stesso tempo, dalle pratiche di produzione e riproduzione delle frontiere. Inoltre, poiché tali pratiche si mobilitano sempre in ambito sociale, provare a identificare dinamiche transfrontaliere in luoghi particolari ci aiuta a riconoscere le forme di ristrutturazione di confini, restrizioni, margini, bordi, controlli e regolazioni e le loro conseguenti modalità di destabilizzare e ristabilizzare soggettività e opportunità di vita.

Inoltre, il globale e il locale si combinano in contesti glocali di pratiche sociali durature e mutevoli (Jones, 1992; Rodríguez, 1995) che compongono nuovi cronotopi o nuove logiche spazio-temporali. Le reti o 'campi sociali' derivanti, connettono località, stati nazionali, persino continenti, creano spazi culturali e sociali ibridi e mutevoli (Featherstone, 1990; Gupta and Ferguson, 1992; Landolt, Autler, and Baires, 1999). All'interno di questi, i migranti ricostruiscono le loro identità regionali, nazionali, continentali, raziali, etniche, socio-culturali e politiche come forma di adattamento ad una esistenza liquida e multinazionale, dalle molteplici frontiere. I migranti e le persone che vivono attraverso/oltre le frontiere assumono identità multiple, nel momento in cui negoziano le loro posizioni tra e all'interno di culture, stati-nazione e altri contesti delimitati/attraversati da frontiere (Glick Schiller, Basch and Blanc-Szanton, 1992; Glick Schiller and Fouron, 1999; Kearney, 1991; Torres and Momsen, 2005, p. 319).

Le politiche transfrontaliere raffigurano le relazioni sociali come ancorate a (ma anche trascendenti da) particolari culture, stati nazionali e altre dimensioni definite da frontiere (come quelle di razza, etnia, genere, età, capacità fisiche, orientamento sessuale, ideologia politica, lingua, religione, ecc.). Si enfatizza così l'importanza e la continua rielaborazione delle frontiere e dei processi di formazione delle frontiere, delle politiche statali (locali, regionali, nazionali, internazionali) e delle identità individuali, sociali, regionali/nazionali/ continentali. In questo modo è possibile riconoscere come i network della comunicazione e delle pratiche sociali, spesso, li trasgrediscono.

Le diverse mobilità di attori, capitale, informazione, caratteri culturali, beni e le loro intersezioni hanno giocato un ruolo chiave nella costruzione sia dei paesi sia del continente latinoamericano come spazi di frontiera mobili e dinamici. Arturo Escobar dice: «è fondamentale riconoscere che l'America Latina è oggi una realtà globale - l'America Latina è letteralmente il mondo intero» (Escobar, 2006, p. 13). Di conseguenza, un processo continuo di (ri)costruzione di luoghi ed identità mol- 
teplici, in tutto il mondo, si manifesta in diversi gradi di liminalità, ibridazione e sincretismo che includono, ma allo stesso tempo superano, quelli che sono generalmente riconosciuti come i tratti distintivi dell'America Latina. Allo stesso modo, le identità d'individui, collettivi e luoghi latino-americani, pur mantenendo, a diversi gradi un nucleo latino-americano generalmente riconoscibile, spesso diventano più flessibili, performativi, permeabili e ne trascendono le frontiere.

Il Latin Lab esplora la produzione e trasformazione di tipi di spazio nuovi e convenzionali in America Latina, le identità socio-culturali e politiche e le articola in un quadro transfrontaliero, in un'arena transnazionale. Vogliamo comprendere i diversi sotto-ambiti di vita e azione attraverso e oltre le frontiere, in cui diversi soggetti sono impegnati a verificare la loro efficacia individuale, collettiva, istituzionale e socio-spaziale e le loro implicazioni. Prestiamo particolare attenzione al modo in cui le popolazioni sottomesse resistono, si adattano e coproducono dinamiche e progetti transnazionali e attraverso le frontiere, rivolti a se stessi e/o alle proprie comunità e al processo attraverso cui tali soggetti transfrontalieri - occupando differenti posizioni 'qui e altrove' e componendo altri 'qui e altrove' - ne sono riplasmati. Vogliamo indagare gli effetti sulle condizioni di conoscenza, potere, soggettività, e spazio che queste dinamiche producono (Irazábal, 2009) e riflettere sui loro attuali o potenziali contributi a un'ulteriore oppressione o emancipazione.

Gli studiosi di America Latina e di altre aree in tutto il mondo stanno esaminando questi complessi fenomeni in molteplici contesti e scale, ma le loro intuizioni e ricerche devono ancora essere presi in considerazione da una prospettiva collettiva. Transbordering Latin Americas mette in dialogo creativo ricercatori provenienti dalle 'scienze spaziali' - architettura, conservazione, urban design, urbanistica e geografia - e altri settori complementari - antropologia, storia, economia, relazioni internazionali e sociologia. Come Latin Lab esploriamo la latino-americanità in tutto il mondo e integriamo all'interno degli studi latino-americani, prospettive teoriche e metodologiche basate sull'interazione tra scienze spaziali e altri campi, contribuendo così al settore innovativo degli studi di frontiera. Jackson, Crang e Dwyer osservano come spesso gli studi transnazionali abbiano «sottovalutato le trasformazioni dello spazio» $(2004$, p. 1).

Il dialogo multidisciplinare tra architettura, conservazione, urban design, urbanistica e geografia e altre discipline nonspaziali può sovvertire questa condizione. È in questo modo che abbiamo promosso una nozione espansiva di spazio transbordering - dove pratiche sociali in trasformazione evocano nuove spazialità e vice-versa - per migliorare la nostra conoscenza della latino-americanità. Tenendo a mente questa nozione di spazio transbordering, la nostra analisi delle costruzioni sociali in America Latina soggette a queste dinamiche, rappresenta contemporaneamente un progetto teorico e politico, che mira a contribuire ad una più profonda delucidazione dei suoi impatti sulla produzione di politiche, di spazio, sulla ricerca e sull'insegnamento. L'intento non è, dunque, solo quello di offrire casi studio rivelatori e promuovere la ricerca teorica e transdisciplinare, ma anche di assistere il pensiero creativo e progressista negli ambiti della politica, della ricerca e della pedagogia.
Cosa intendiamo per 'Transbordering Latin Americas'? Transbordering Latin Americas esplora le condizioni socioculturali e spaziali di un'America Latina transnazionale, al di là delle proprie frontiere, in tutto il mondo, e attraverso varie scale: dal corpo visto da una prospettiva di genere e di razza, fino all'arena nazionale e transnazionale. Quando usiamo il plurale Americhe Latine, rifiutiamo volutamente la nozione di un'America Latina unificata, coesa e statica e una corrispondente identità singolare - un modo di essere o di essere percepiti come latino-americani. La frase 'transbordering Latin Americas' comprende quindi istanze di ciò che può essere definito come 'latino-americano' (che è, a sua volta, aperto al dibattito e alla trasformazione), che accade tramite relazioni sociali pluri-locali - esistenti all'interno, tra, e al di sopra del contenitore spaziale tradizionale delle società nazionali e continentali, senza 'madrepatrie' chiare o definite (Pries, 2004; Irazábal, 2012). Americhe Latine, al plurale, intende spingere oltre la problematizzazione di un 'nazionalismo metodologico', o la tendenza a legare società e stato nazionale. Infatti, nonostante la sua innegabile e continua importanza nell'inquadrare dinamiche sociali, lo stato-nazione è stato contestato come 'unità' naturale del mondo moderno e in particolare come (elemento) utile per studiare $i$ fenomeni migratori e diasporici (Duany, 2011; Wimmer and Glick Schiller, 2002). Suggeriamo la necessità di andare oltre non solo il 'nazionalismo metodologico', ma anche il 'continentalismo metodologico' per decostruire e ricostruire efficacemente una nozione di America Latina plurale e dinamica.

Quando ci riferiamo a luoghi liminali, culture e poteri nel sottotitolo del libro, ci scontriamo con le fasi e le condizioni transitorie e instabili di soglie sensoriali e mentali e la natura intermedia dei vari luoghi, culture e poteri che studiamo. Vogliano indicare, inoltre, la natura complessa e mobile di luoghi, culture e poteri e intendiamo esplorarne i processi di mutua rappresentanza. Il termine ambiguo ( $T$ ) here (qui e altrove) riflette nuovi cronotopi o composizioni spazio-temporali che non sono completamente qui o altrove, ma sono contemporaneamente qui e altrove. Il termine allude, inoltre, alla crescente difficoltà nel distinguere tra tempo e spazio - ciò che alcuni teorici hanno nominato compressione spazio-temporale della condizione postmoderna (Harvey, 1990) e geografie di temporalità o «SpazioTempo» (May and Thrift, 2001). Nel comprendere questa condizione e nell'incorporarla nella nostra analisi, la nozione di $(T)$ here tende contemporaneamente ad evidenziare la dimensione spaziale dei luoghi in cui soggetti di frontiera si muovo e abitano, suggerendo al contempo che questi luoghi sono nuovi assemblaggi di 'qui' e 'altrove' che si diffondono pluri-localmente, luoghi che possiedono frammenti di 'altrove' incorporati nei loro effettivi 'qui'.

\section{Spazi, culture e potere liminali 'qui e altrove': perché occuparsene?}

Il nostro lavoro riflette e assume (alcune) domande poste da Miraftab e Salo (2012) come questioni critiche attorno alle quali proporre un'agenda rinnovata e nuovi quesiti per l'insegnamento della pianificazione, la produzione di spazio e di politiche. Questa sezione trae dalle nostre ricerche più recenti alcuni casi studio in America Latina, che affrontano queste domande da diverse prospettive. 
Quali sono le nuove spazialità delle città in un mondo sempre più diseguale e caratterizzato dalla presenza di frontiere? Come cambiano attraverso le aree urbane e le regioni?

Entrambi i casi presentati di seguito, relativi alla produzione industrializzata di abitazioni in aree extra-urbane e a megaprogetti di terziario in zone urbane centrali in Messico, illustrano le controversie che coinvolgono, da un lato, progetti di sviluppo governativi e imprenditoriali, e dall'altro l'adattamento e la sovversione da parte delle comunità interessate e le conseguenti trasformazioni sociali. Le ideologie della modernità, del progresso, del nazionalismo e della globalizzazione supportano versioni particolari di un'urbanistica neo-liberista che, in questi due distinti casi messicani, vengono sia respinte sia sostenute, illustrando le spazialità emergenti e rapidamente proliferanti di un mondo attraversato da frontiere.

In primo luogo, Cristina Inclán-Valadez (2014) esplora la diffusione, in anni recenti, d'insediamenti residenziali a basso costo e a larga scala. Analizza in particolare la creazione di piccoli centri urbani residenziali per la classe media in Messico, esaminando il fenomeno di Casas Geo (il più grande operatore privato nel campo dell'edilizia a basso costo in America Latina) nella città di Cuernavaca. Il Messico costruisce più di $\mathbf{5 0 0 . 0 0 0}$ abitazioni all'anno nelle periferie profonde delle città consolidate. Queste abitazioni, a schiera e organizzate in raggruppamenti recintati e con un disegno viabilistico uniforme, sono rivolte a famiglie della classe media e bassa, con una specifica iconografia borghese. Inclán-Valadez dimostra che, piuttosto che essere semplicemente il prodotto 'finito' di una decisione progettuale, questi schemi abitativi evolvono attraverso la partecipazione di un'ampia gamma di attori, inclusi visitatori, arredatori, agenti immobiliari, costruttori, e residenti attuali e potenziali. Tali schemi intendono rappresentare una formula per una crescita urbana 'buona', un formato legittimato per la produzione di abitazioni e per creare città 'modello'. Vengono, quindi, promossi per formulare le condizioni di miglioramento sociale di milioni di famiglie messicane. Inclán-Valadez esplora come un modello globale di 'città sorte dal nulla' è interpretato e vissuto localmente dai residenti di un particolare complesso Geo-Bosques. Identifica le caratteristiche generiche che possono essere individuate nella produzione dello schema Casas Geo e discute di come il modello sia stato costruito, sviluppato, organizzato e invariabilmente contestato a seguito di continue strategie locali impiegate da differenti attori (per lo più residenti). L'obiettivo di queste azioni è di raggiungere una configurazione socio-spaziale 'ideale' che cerchi di emulare le nozioni globali idealizzate di uno stile di vita borghese ed incrementare il senso di sicurezza e lo status sociale dei residenti. Questo esempio illustra come l'esperienza di nuovi schemi abitativi locali prende forma attraverso la 'vernacolarizzazione' di referenti globali e genera, quindi, la creazione di nuovi paesaggi transnazionali in Messico, spesso con conseguenze spaziali, funzionali e sociali critiche.

In secondo luogo, María Moreno Carranco (2014) ci conduce a Città del Messico per discutere l'emergere di 'megaprogetti urbani' come una delle strategie dominanti nella costruzione delle città messicane durante l'attuale congiuntura neoliberista e l'apertura dell'economia messicana. Città del Messico si trova in competizione con altre città, come Miami e São Paulo, per diventare un nodo metropolitano sempre più importante nelle reti finanziarie e produttive dell'economia globale $\mathrm{o}$, almeno, regionale. Moreno Carranco si concentra sul megaprogetto di Santa Fe, il più grande piano di sviluppo urbano in America Latina, ampiamente criticato per essere l'esito di una pianificazione transnazionale imposta attraverso meccanismi non-democratici a beneficio del capitale globale e delle élite locali. Il progetto di Santa Fe non risulta essere adeguatamente integrato con il suo contesto locale, a causa dell'ambizione di creare un 'luogo globale' che incarni le caratteristiche fisiche necessarie per attrarre le compagnie multinazionali e per migliorare la posizione di Città del Messico sulla scena globale. Lo studio analizza le nuove geografie e le dissonanze culturali che emergono in questo sforzo di competere per una migliore posizione nell'arena delle città globali. Lo scollamento tra le promesse del megaprogetto e la realtà effettiva di Santa Fe ha prodotto condizioni urbane paradossali in cui i residenti stanno sopperendo all'inadeguata manutenzione dell'area da parte del governo. Questa situazione sta aggravando ulteriormente la crescente segregazione sociospaziale, l'esclusione, la gentrificazione, la privatizzazione dello spazio della città, e forme alternative di governance. Mentre compagnie multinazionali, edifici classe AAA, e residenze di lusso si scontrano, a Santa $\mathrm{Fe}$, con alcune condizioni urbane simili a quelli degli insediamenti urbani irregolari, i discorsi del marketing enfatizzano quegli stessi elementi che scarseggiano nel megaprogetto, vendendo un immaginario luogo globale disconnesso dalla sua realtà. L'analisi del mega-progetto di Santa Fe illustra questi paradossi e come le pratiche locali siano una parte costituiva di questo spazio globale immaginato, trasformato dalla produzione continua di nuovi comportamenti e nuove forme di appropriazione dello spazio urbano.

Quali sono le forme, nuove o persistenti, di disuguaglianza che questi processi producono, in particolare riguardo alle questioni di genere, razza, salario, alle condizioni abitative, di sicurezza, violenza, status legale, ecc.? Quali implicazioni hanno i processi di produzione ed attraversamento delle frontiere nella produzione di condizioni inique mutevoli e spesso crescenti?

I discorsi e le pratiche del capitalismo e del neoliberismo vengono spesso incorporati dai media dominanti, dai regimi economici (compreso la loro criminosità e violenza), e dai regimi di politiche migratorie; e spesso sono (entusiasticamente) adottati e talvolta sovvertiti da chi vi è sottoposto. Gli immaginari e le politiche identitarie attorno alla nozione di barrio, per esempio, aiutano a mostrare le tensioni tra costruzioni egemoniche e antiegemoniche di genere, così come l'intersezione dei loro tratti identitari nazionali, di classe, etno-raziali e di età. Tali tensioni mettono in dubbio gli ordini socio-economici ed istituzionali, e potenzialmente aprono ad opportunità impreviste per l'autorappresentazione e l'emancipazione di individui e popolazioni assoggettate.

Yves Pedrazzini (2014), per esempio, ci offre il corpo umano maschile come luogo della lotta tra formazioni identitarie individuali e globali. Pedrazzini spiega come il barrio, luogo comune urbano e latino-americano, sia entrato nella sfera dei media internazionali, percepito generalmente come una giungla di asfalto, infestata di barbari. La particolare 'latinità' del barrio è replicata a scala globale, spesso nei suoi tratti peggiori: nelle rappresentazioni comuni, strade buie abbandonate a spacciatori e adolescenti armati sostituiscono l'immagine di quartieri popolari ricchi di risorse e più creativi. Nessuna di queste immagini è 


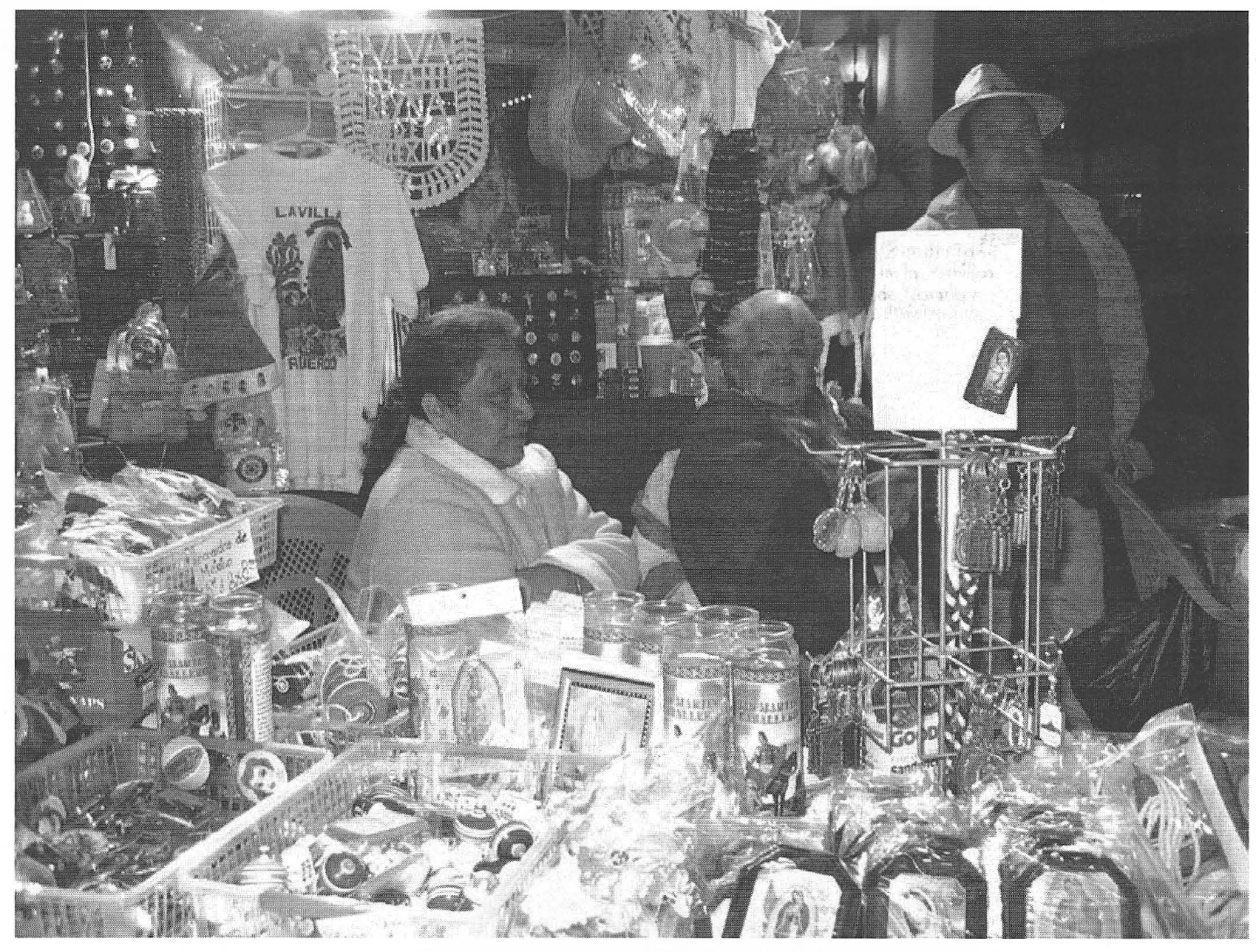

Venditrici di souvenir del villaggio della Vergine di Guadalupe (Città del Messico) in Plaza

Mexico a Los Angeles, 2005

Fonte: foto di G. Fumero 
egemonica, ma la visione inquietante di oggi supera spesso la scena pittoresca di ieri. La globalizzazione di un'estetica della violenza, fondata sulla retorica dei 'poveri e cattivi' e del 'quartiere pericoloso' chiude le persone in un immaginario globale dell'America Latina ristretta a due figure estreme, il criminale e l'animale da party: una essenzializzazione dalle drammatiche conseguenze. La società dello spettacolo riproduce queste immagini in un 'racconto' continuo della città latino-americana, presentandola come un luogo disordinato che intimorisce, ma anche seduce ed eccita. Pedrazzini esamina come la costruzione del 'mito del quartiere violento' e la demonizzazione del gangster (il malandro in slang venezuelano) attribuiscano la responsabilità dei fenomeni urbani negativi al giovane e povero maschio latino e alla sua cultura popolare. Tale attribuzione permette l'abbandono di responsabilità da parte della società, in condizioni strutturali che creano e riproducono l'impoverimento delle classi lavoratrici. Di conseguenza, una liminalità negativa viene creata attorno ad ogni 'spazio latino' (quartiere, strada, bar) che ostacola la possibilità che 'latino-americanismi' positivi incoraggino legami sociali con e oltre la diaspora latina. Pedrazzini critica i paradossi e le ipocrisie di questa costruzione del maschio latino nel cosiddetto 'nord globale'.

Quali sono $i$ modi, ampiamente iniqui nelle loro condizioni di vita, in cui le persone negoziano la propria sopravvivenza, sicurezza e dignità in questi spazi (urbani, suburbani, extraurbani e rurali)? Come gli abitanti di questi insediamenti emergenti, in crescita o in trasformazione, reclamano e affermano il loro diritto al sostentamento e alla dignità? Come praticano il loro diritto alle città e alla cittadinanza?

I luoghi percorsi dal turismo transnazionale sono spesso siti favorevoli all'analisi delle relazioni tra le forme della soggettività umana e le pratiche di negoziazione nello spazio. I casi studio coinvolgono luoghi a diverse scale, tra cui una città (Cusco, Peru) e un centro commerciale (Plaza Mexico, Stati Uniti). Questi (spazi) agiscono come modelli di paesaggi turistici e multiculturali (Cartier and Lew, 2005; Irazábal, 2006) che permettono una riteorizzazione delle relazioni tra turisti e viaggio, 'viaggiatori' e 'nativi', e i contesti e le implicazioni socioculturali, spaziali e politiche di questi processi e formazioni in un'epoca caratterizzata dalla presenza di molteplici frontiere. Miriam Chion (2014) presenta Cusco come una delle città latino-americane di piccole dimensioni maggiormente coinvolte da processi transnazionali. Cusco accoglie uno dei più alti numeri di turisti per residente in tutto il continente. Riceve inoltre numerosi investimenti esteri e di organizzazioni internazionali e possiede una popolazione sempre più diversificata. Dal periodo degli Inca, centro politico di un ampio territorio di comunità indigene, all'epoca attuale uno dei maggiori centri turistici dell'America Latina, Cusco è un esempio sia della forza che della malleabilità della cultura locale nella sua interazione con un'ampia varietà di influenze transnazionali economiche e culturali. In alcuni casi, nella città, musicisti tradizionali e artigiani operano separati dalle attività contemporanee e commerciali, mentre in altri casi sono connessi ad esse. Queste pratiche culturali esemplificano la produzione di spazi e soggetti di frontiera, anche negli ambiti più tradizionali dell'artigianato rurale, e la dissoluzione del divario stabile tra locale e internazionale, dislocato/dislocante e tradizionale/ moderno. Data la sua ricca storia e la pressione del turismo contemporaneo, Cusco fornisce un contesto di intenso sviluppo in cui queste complessità sono amplificate. Il concetto di 'città della memoria' proposto da Sandercock e il discurso andino moderno ('il discorso andino moderno') di Nieto inquadrano l'analisi della produzione di questi spazi tramite l'intreccio di una forte conoscenza tradizionale e di un senso di identità, che coinvolgono processi di produzione e riproduzione sociale ed espandono i flussi di apprendimento.

Clara Irazábal e Macarena Gómez-Barris (2014) discutono delle nuove dinamiche del turismo e delle loro implicazioni per l'identità e lo sviluppo di comunità nell'area metropolitana di Los Angeles a Plaza Mexico, un centro commerciale. Di concezione e proprietà d'investitori coreani, Plaza Mexico incarna un caso unico di invenzione e mercificazione delle tradizioni, per migranti insediatisi nel contesto locale e cittadini statunitensi di origini messicane/latine. La piazza è un collage architettonico d'icone regionali e nazionali messicane che trasportano i suoi avventori 'in Messico' (fig. 1). Nello spostamento da e nella migrazione verso/all'interno degli Stati Uniti, questi avventori (come i loro antenati) hanno subito diversi processi di deterritorializzazione e riterritorializzazione delle proprie identità, delle loro pratiche abitative, e delle proprie immagini del luogo di origine. Plaza Mexico fa leva su questi immaginari per produrre uno spazio di turismo legato alla diaspora, per mezzo del quale il capitalismo reinventa opportunisticamente la tradizione, all'interno di un contesto strutturale in cui la mobilità migrante rimane limitata/confinata. Molti visitatori non hanno le risorse economiche e legali per recarsi in Messico - anche se vorrebbero - e quindi vivono la plaza come un suo surrogato disponibile. Essi, tuttavia, si appropriano dello spazio in modi che permettono la celebrazione e la reinvenzione delle tradizioni, l'espansione della cittadinanza (attraverso classi d'inglese e di cittadinanza, fiere culturali e della salute e simili), e la mobilizzazione di uno sviluppo transnazionale (attraverso associazioni patriottiche, aiuti a popolazioni disastrate e catene di prodotti che uniscono la Plaza con i luoghi d'origine dei visitatori). Mentre molte teorie contemporanee del turismo, del viaggio e del luogo enfatizzano l'erosione dei confini nazionali e la fluidità dei territori, il caso di Plaza Mexico ci porta ad apprezzare questi fenomeni e, allo stesso tempo, i loro opposti - il rafforzamento dei confini nazionali e il loro impatto sulla (im)mobilità di milioni di persone. Luoghi come Plaza Mexico influiscono e intensificano questi processi e inoltre aprono nuove opportunità per lo sviluppo di comunità in un'arena transfrontaliera.

Quali sono le modalità di intervento attraverso politiche formali o pratiche informali praticate da funzionari pubblici, attivisti e abitanti per affrontare le sfide urbane emergenti o persistenti? Esistono maniere radicalmente differenti per rispondere a questa domanda in diversi contesti multi-scalari, dall'esplorare la condizione specifica delle comunità indigene in America Latina che formano nazioni contese all'interno di nazioni e come queste formazioni sono spesso costruite, supportate e contestate in arene transnazionali (Tovar-Restrepo, 2014); ai processi specifici attraverso i quali i gruppi diasporici di latinoamericani, che vivono all'estero, creano per se stessi spazi e senso di appartenenza nei loro luoghi di origine e di adozione. 
Faranak Miraftab (2014) esamina le esperienze multiculturali dell'immigrazione a Bearstown, Illinois. Bearstown è una comunità multiculturale in crescita nel cuore degli Stati Uniti. Una piccola città del Midwest che ha ricevuto un rapido afflusso sia di latini che di africani occidentali, a causa delle pratiche di reclutamento di manodopera della sua industria di confezionamento della carne. Mentre gli studi classici sull'immigrazione tendono a focalizzare l'attenzione su un singolo gruppo di migranti e sulle dinamiche di contrapposizione con la popolazione nativa dominante, lo studio di Miraftab esamina produttivamente la relazione intima ed iniqua che connette la rivitalizzazione di questa cittadina, dedita al confezionamento, ai processi di sviluppo nelle comunità originarie dei lavoratori migranti in Togo e in Messico. Per cogliere l'azione dei migranti nel negoziare le proprie esperienze di migrazione in un ambiente nuovo e problematico, lo studio evidenzia come le famiglie, gli amici e le istituzioni locali dei migranti sovvenzionino la crescita demografica e la riproduzione di luoghi nelle comunità di destinazione dei migranti, una 'ristrutturazione globale della riproduzione sociale'. Questo studio non solo getta nuova luce sulla nostra comprensione delle geografie multiculturali emergenti e sullo sviluppo locale basato sull'immigrazione in Messico, Togo e negli Stati Uniti, ma rende inoltre visibili le interconnessioni globali nei processi di espoliazione e sviluppo, e ci aiuta a registrare i nuovi corsi di un'azione politica basata sulla comunità che può supportare un'integrazione demografica sana in luoghi in rapido cambiamento.

Rosa Cervera (2014) ci conduce attraverso la 'archicultura' dell'immigrazione a Tetuàn, Madrid. Il fenomeno dell'immigrazione, relativamente nuovo in Spagna, sta producendo una trasformazione, fisica e culturale, a livello urbano e architettonico delle città iberiche, che è ancora poco studiata. Madrid, la capitale della Spagna e il centro urbano economicamente più attivo del paese, ha ricevuto più di 500.000 migranti di origine latino-americana negli ultimi venticinque anni, la maggior parte dei quali negli ultimi quindici. Il caso specifico del quartiere Tetuán-La Ventilla, localizzato nella zona nord di Madrid, è uno degli esempi più interessanti d'insediamento della popolazione latino-americana nella città. A causa dell'espansione urbana, il quartiere è vicino ad alcune delle zone di pregio e più rappresentative di Madrid, incluso un nuovo quartiere degli affari. Tuttavia, la storia di Tetuán-La Ventilla - un quartiere fuori dalle mura della città storica con un'endemica mancanza di pianificazione e una topografia complessa e molto accidentata - ha permesso a questo luogo di rimanere, nonostante la sua localizzazione strategica e le sue potenzialità a livello urbano, un'area marginale e quindi adatta all'insediamento di migranti. Cervera esamina l'impatto dell'immigrazione sulle trasformazioni del quartiere e sulla sua architettura, una trasformazione che è stata condotta, il più delle volte, in maniera spontanea ma silenziosa. Questa modalità di azione, al di fuori della norma, è dovuta soprattutto alla scarsa lungimiranza della pubblica amministrazione e alla mancanza di controllo dell'intero processo, dato il rapido ritmo di insediamento dei migranti. Molte problematiche architettoniche e urbane non vengono affrontate adeguatamente, a causa della cecità di convenienza delle autorità amministrative e della segretezza e dell'impermeabilità della società migrante, che usa questi tratti come strategie di auto-conservazione. Il lavoro di
Cervera rappresenta una riflessione pedagogica. Nel processo d'identificazione e studio delle caratteristiche dell'area, ella presenta la metodologia assunta nel Master in Advanced Project of Architecture and City dell'università di Alcalà e discute di come, all'interno di quel quadro, è stato possibile dare risposte progettuali sensibili alle condizioni sociali, ambientali e urbane del quartiere. Riflette sulle sfide e responsabilità del progettare per questo tipo di comunità transnazionali.

\section{Transbordering Planning: la strada da/verso il qui e l'altrove}

Come gli esempi offerti in questo articolo dimostrano, sia che il focus del nostro lavoro di insegnamento, ricerca, trasformazione di luoghi e politiche sia domestico o internazionale, abbiamo tutti bisogno di accettare i crescenti processi di produzione di frontiere nel nostro mondo e le dinamiche politiche, economiche, socioculturali e spaziali derivanti. Dobbiamo essere consapevoli di queste dinamiche e render conto di esse nelle nostre analisi e proposte. Torres e Momsen ci ammoniscono che «i tentacoli delle forze transnazionali sono di vasta portata e persistenti, estendendosi irresistibilmente per includere anche i più lontani angoli della terra» (Torres and Momsen, 2005, p. 332). Le ramificazioni espansive e non intenzionali di questo progetto - la capacità di ristrutturare aree e comunità apparentemente remote e libere - fornisce un ammonimento per tutte le iniziative di sviluppo. Insito nel nostro studio, ritengo, dovrebbe essere una critica ai processi correnti di sviluppo guidati dal capitalismo globale. Comprendere l'America Latina (o qualsiasi altra regione del mondo) come uno spazio di frontiera, come appunto Transbordering Latin Americas, fornisce visioni del «potere del capitalismo globale che si espande geograficamente, trasforma e mercifica lo spazio, e stringe la propria presa su tutti gli aspetti della vita" (Ibid.). Nel fare questo, la globalizzazione e lo sviluppo capitalista hanno spesso «esacerbato le disuguaglianze esistenti e creato nuove inique geometrie di potere a diverse scale.

Queste iniquità coinvolgono non solo il potere e l'economia, ma sono anche evidenti nella subordinazione delle culture, delle strutture sociali e degli ambienti locali» (Ibid.).

Gli esempi di Transbordering Latin Americas, qui discussi, illustrano la complessa rete di attori e relazioni sociali che esistono a diverse scale costruendo spazi che spesso riproducono ineguaglianze e ne creano di nuove tra persone, comunità, regioni e nazioni. D'altro canto, esse indicano finestre per nuove opportunità, sebbene frugali e difficili da ottenere o sostenere, che potrebbero promuovere l'empowerment, la sostenibilità e la giustizia individuale e collettiva.

Nell'esaminare criticamente il ruolo delle forze transnazionali, nel riplasmare le realtà locali dentro l'America Latina - 0 altrove - e nel promuovere un cambiamento progressista, potrebbe essere possibile cogliere meglio l'affermazione del World Social Forum che «un altro mondo è possibile», caratterizzato da uno sviluppo più equo e sostenibile e da maggiori spazi di solidarietà, conoscenza emancipatrice, reti di potere, e soggettività realizzate (Irazábal, 2009). Se un altro mondo è possibile, allora altre Americhe Latine - Asie, Afriche, Europe - sono possibili, accogliendo tutti al di là e attraverso le frontiere.

(traduzione dall'inglese di Alice Buoli) 


\section{Nota del traduttore}

Transbordering sia nella sua forma aggettivale sia nella sua declinazione verbale progressiva, non ha un corrispettivo letterale nella lingua italiana. Per questo motivo, nella traduzione del saggio di Clara Irazábal, espressioni come 'transborder', 'transbordering', 'transborderism', che riguardano processi e pratiche che avvengono attraverso, oltre che a causa, della presenza alle diverse scale di molteplici frontiere (politiche, socio-culturali, economiche e simboliche), sono state tradotte variamente con diverse perifrasi al fine di cogliere il significato attribuito dall'autrice a questi termini. In alcune occasioni, l'aggettivo 'transfrontaliero' o 'transnazionale' (quando coincidente) e l'espressione 'di frontiera' sono stati utilizzati per necessità di sintesi o perché maggiormente coerenti nel contesto della frase. Si è scelto quindi di mediare tra chiarezza del testo italiano e mantenimento del significato originale dei termini utilizzati dall'autrice. Altrove ancora, come nel titolo del saggio e in alcuni passaggi fondamentali dell'articolo, si è scelto di mantenere, per chiarezza, l'originale in inglese. Inoltre si segnala che tutte le citazioni sono state tradotte dall'originale in inglese.

\section{Note}

1. L'articolo è stato sottoposto a Territorio in occasione del seminario «The Medellin Experience: Research and Practice across Urban Planning and Design», organizzato da Davide Ponzini presso il Politecico di Milano nel Gennaio 2014.

2. Clara Irazábal è Direttrice del Latin Lab e Assistant Porfessor of Urban Planning presso la Graduate School of Architecture, Planning, and Preservation della Columbia University (New York).

3. $N d t$ : originale in inglese.

4. Il Latin Lab fornisce una piattaforma per inzitive di ricerca, educative e di servizio relativa all'architettura e alla pianificazione in America Latina e nei paesi Caraibici (Lac). Con sede presso la Graduate School of Architecture, Planning and Preservation (Gsapp) della Columbia University, il Lab mira a divenire un punto di riferimento nello studio dell'ambiente costruito e dello sviluppo di comunità nei paesi Lac e delle sue diaspore, e una risorsa primaria per contribure ad una loro trasformazione equa e sostenibile. Le principali linee di lavoro del Lab sono Migrazioni ed Etno-Urbanistica, Resilienza e Upgrading Urbano, Pianificazione Regionale e Transnazionale.

5. Il libro è basato sugli esiti della conferenza "Transnational Latin Americanisms: Liminal Places, Cultures, and Powers ( $T$ ) Here» tenutasi presso la Columbia Univerisity il 4-5 marzo del 2010. Prima Conferenza della serie Urban Latin American Series. Columbia University, 4-5 marzo del 2010 (www.youtube.com/user/columbiauniversity/videos?query = Transnational + Latin + Americanisms).

\section{Riferimenti bibliografici}

Appadurai A., 1996, Modernity at Large: Cultural Dimensions of Globalization, University of Minnesota Press, Minneapolis.

Bada X., 2010, «Mexican Migrants: The Attractions and Realities of the United States», Latin American Research Review, vol. 45, n. 2, pp. 236-244, doi: 10.1353/lar.2010.0004.

Bauder H., 2013, «Nation, 'Migration' and Critical Practice», Area, vol. 45, n. 1, pp. 56-62, doi: 10.1111/j.1475-4762.2012.01129.x.

Cartier C. and Lew A.A., 2005, eds., Seductions of Place: Geographical Perspectives on Globalization and Touristed Landscapes, Routledge, Oxford, UK.

Castells M., 2004, «An Introduction to the Information Age», in Webster F. et al. (eds.), The Information Society Reader, Routledge, London and New York, pp. 138-149.

Cervera R., 2014, «The Archiculture of Immigration in the Borough of Tetuán (Madrid): A Pedagogical Approach», in Irazábal C. (ed.), Transbor- dering Latin Americas: Liminal Places, Cultures, and Powers (T)Here, Taylor \& Francis Group, Routledge, New York, London, pp. 278-301.

Chion M., 2014, "Cusco: City of Memory», in Irazábal C. (ed.), Transbor dering Latin Americas: Liminal Places, Cultures, and Powers (T)IIere, Taylor \& Francis Group, Routledge, New York, London, pp. 79-95.

Crang P., Dwyer C. and Jackson P., 2003, «Transnationalism and the Spaces of Commodity Culture", Progress in Human Geography, vol. 27, pp. 438-456, doi: 10.1191/0309132503ph4430a.

Duany J., 2011. Blurred Borders: Transnational Migration Between the Hispanic Caribbean and the United States, The University of North Carolina Press, Chapel Hill, NC.

Escobar A., 2006, «Revisioning Latin American and Caribbean Studies: A Geopolitics of Knowledge Approach", Latin American Studies Association Forum, vol. 2, pp. 11-13.

Featherstone M., 1990, «Global Culture: Nationalism, Globalization and Modernity», Theory, Culture and Society, vol. 7, pp. 1-14, doi: $10.1177 / 026327690007002001$.

Glick Schiller N. and Fouron G.E., 1999, «Terrains of Blood and Nation: Haitian Transnational Social Fields», Ethnic and Racial Studies, vol. 22, n. 2, pp. 340-366, doi: 10.1080/014198799329512.

Glick Schiller N., Basch L. Blanc-Szanton C., 1992, «Transnationalism: A New Analytic Framework for Understanding Migration», in Id. (eds.), Towards a Transnational Perspective on Migration: Race, Class, Ethnicity, and Nationalism Reconsidered, The New York Academy of Sciences, New York, pp. 1-24.

Gupta A. and Ferguson J., 1992, "Beyond 'Culture': Space, Identity, and the Politics of Differencen, Cultural Anthropology, vol. 7, n. 1, pp. 6-23, doi: $10.1525 /$ can.1992.7.1.02a00020.

Harvey D., 1990, The Condition of Postmodernity: An Enquiry into the Origins of Cultural Change, Blackwell, Cambridge, MA.

Inclán-Valadez C., 2014, «Building New Geographies in Urban Mexico: The Case of the Casas Geo», in Irazábal C. (ed.), Transbordering Latin Americas: Liminal Places, Cultures, and Powers (T)Here, Taylor \& Francis Group, Routledge, New York, London, pp. 166-186.

Irazábal C., 2005, City Making and Urban Governance in the Americas: Curitiba and Portland, Ashgate, London.

Irazábal C., 2006, «Seductions of Place: Geographical Perspectives on Globalization and Touristed Landscapes", Regional Studies, vol. 40, n. 7 , pp. $805-806$.

Irazábal C., 2008, ed., Ordinary Places, Extraordinary Events: Citizenship, Democracy, and Public Space in Latin America, Taylor \& Francis Group, Routledge, New York, London.

Irazábal C., 2009, «Realizing Planning’s Emancipatory Promise: Learning from Regime Theory to Strengthen Communicative Action», Planning Theory, vol. 8, n. 2, pp. 115-139, doi: 10.1177/1473095209102230.

Irazábal C., 2011, «Ethnoscapes», in Banerjee T. and Loukaitou-Sideris A. (eds.), Companion to Urban Design: Routledge Companions, Taylor \& Francis Group, Routledge, London and New York, pp. 562-573.

Irazábal C., 2012, "Transnational Planning: Reconfiguring Spaces and Institutions", in Krätke S., Wildner K. and Lanz S. (eds.), Transnationalism and Urbanism, Taylor \& Francis Group, Routledge, London and New York, pp. 72-90.

Irazábal C., 2014, "Introduction: What Do We Mean by 'Transbordering Latin Americas'?», in Id. (ed.), Transbordering Latin Americas: Liminal Places, Cultures, and Powers (T)Here, Taylor \& Francis Group, Routledge, London and New York, pp. 1-19.

Irazábal C. and Gómez-Barris M., 2014, «Diasporic Tourism: Immigrant Politics and Place Making in Los Angeles's Plaza Mexicon, in Irazábal (ed.), op.cit., pp. 96-126.

Jackson P., Crang P. and Dwyer C., 2004, «Introduction: The Spaces of Transnationality», in Id. (eds.), Transnational Spaces, Routledge, London, pp. 1-23.

Jones D., 1992, "Which Migrants? Temporary or Permanent», in Glick Schiller, Basch and Blanc-Szanton (eds.), op. cit., pp. 217-224.

Kearney M., 1991, «Borders and Boundaries of State and Self at the End of Empire», Journal of Historical Sociology, vol. 4, n. 1, pp. 52-74, doi: 10.1111/j.1467-6443.1991.tb00116.x. 
Landolt P., Autler L. and Baires S., 1999, «From hermano lejano to hermano mayor: The Dialectics of Salvadoran Transnationalism», Ethric and Racial Studies, vol. 22, n. 2, pp. 290-315, doi: $10.1080 / 014198799329495$.

Levitt P., 2001, «Transnational Migration: Taking Stock and Future Directions", Global Networks, vol. 1, n. 3, pp. 195-216, doi: 10.1111/14710374.00013.

May J. and Thrift N., 2001, «Introduction», in Id. (eds.), TimeSpace: Geographies of Temporality Routledge, New York, pp. 1-46.

Miraftab F., 2014, «Faraway Intimate Development: Global Restructuring of Social Reproduction», in Irazábal (ed.), op. cit., pp. 241-257.

Miraftab F. and Salo K., 2012, Cities and Inequalities in a Transnational World, Joint Area Centers Symposium, March 1-3, Levis Faculty Center, University of Illinois, Urbana-Champaign (http://cgs.illinois. edu/jacs2012).

Moreno Carranco M., 2014, "Global Mexico under Construction: The Santa Fe Megaproject in Mexico City», in Irazábal (ed.), op. cit., pp. $187-214$.

Ong A., 1999, Flexible Citizenship: The Cultural Logics of Transnationality, Duke U.P., Durham (NC) and London.

Pedrazzini Y., 2014, "'The Gang of the Barrio': Invention and Negative Transnationalization of a Latin American Figure of Urbanity", in Irazábal (ed.), op. cit., pp. 61-76.

Portes A., Guarnizo L.E. and Landolt P., 1999, "The Study of Transnationalism: Pitfalls and Promise of an Emergent Research Field», Ethnic and Racial Studies, vol. 22, n. 2, pp. 217-237, doi: $10.1080 / 014198799329468$.

Pries L., 2004, «Transnationalism and Migration: New Challenges for the Social Sciences and Education», in Luchtenberg S. (ed.), Migration, Education and Change, Taylor \& Francis, Routledge, London and New York, pp. 15-39.
Pries L., 2005, "Configurations of Geographic and Societal Spaces: A Sociological Proposal between 'Methodological Nationalism' and the 'Spaces of Flows», Global Networks: A Journal of Transnational Affairs, vol. 5, n. 2, pp. 167-190, doi: 10.1111/j.1471-0374.2005.00113.x.

Rodríguez N.P., 1995, «The Real "New World Order": The Globalization of Racial And Ethnic Relations in the Late Twentieth Century", in Smith P.M. and Feagin J.R. (eds.), The Bubbling Cauldron: Race, Ethnicity and Urban Crisis, University of Minnesota Press, Minneapolis, pp. 211-225.

Roudometof V., 2005, "Transnationalism, Cosmopolitanism and Glocalization", Current Sociology, vol. 53, n. 1, pp. 113-135, doi: $10.1177 / 0011392105048291$.

Smith M.P., 2001, Transnational Urbanism: Locating Globalization, Blackwell, Malden, MA.

Stephen L., 2007, Transborder Lives: Indigenous Oaxacans in Mexico, California, and Oregon. Duke U.P., Durham, NC.

Torres R.M. and Momsen J.D., 2005, «Gringolandia: The Construction of a New Tourist Space in Mexico», Annals of the Association of American Geographers, vol. 95, n. 2, pp. 314-335, doi: 10.1111/j.14678306.2005.00462.x.

Tovar-Restrepo M., 2014, «Nations within Nations: Transnationalism and Indigenous Citizenship in Latin America», in Irazábal (ed.), op. cit., pp. 149-165.

Trotz A.D., 2006, «Rethinking Caribbean Transnational Connections: Conceptual Itineraries», Global Networks: A Journal of Transnational Affairs, vol. 6, n. 1, pp. 1-59, doi: 10.1111/j.1471-0374.2006.00132.x.

Wimmer A. and Glick Schiller N., 2002, «Methodological Nationalism and Beyond: Nation-State Building, Migration and the Social Sciences», Global Networks, vol. 2, n. 4, pp. 301-334, doi: 10.1111/14710374.00043 . 